\title{
Development of a Clinical Scoring System for the Diagnosis of Smear-Negative Pulmonary Tuberculosis
}

\author{
Alonso Soto ${ }^{1,2}$, Lely Solari ${ }^{2}$, Juan Agapito ${ }^{2}$, Carlos Acuna-Villaorduna ${ }^{2}$, \\ Marie-Laurence Lambert ${ }^{3}$, Eduardo Gotuzzo ${ }^{4}$ and Patrick Van der Stuyft ${ }^{3}$ \\ ${ }^{1}$ Hipólito Unanue National Hospital; ${ }^{2}$ Cayetano Heredia University of Peru;Lima, Peru; ${ }^{3}$ Epidemiology and Disease Control Unit, Institute of \\ Tropical Medicine; Antwerp, Belgium; ${ }^{4}$ Tropical Medicine Institute Alexander von Humboldt; Bogota, Colombia
}

\begin{abstract}
This study developed a clinical score based on clinical and radiographic data for the diagnosis of smear-negative pulmonary tuberculosis (SNPT). SNPT was defined as a positive culture in Ogawa in a patient with two negative sputum smears. Data from patients admitted to the emergency ward with respiratory symptoms and negative acidfast bacilli (AFB) smears was analyzed by means of logistic regression to develop the predictive score.Two hundred and sixty two patients were included. Twenty patients had SNPT. The variables included in the final model were hemoptysis, weight loss, age $>45$ years old, productive cough, upper-lobe infiltrate, and miliary infiltrate. With those, a score was constructed. The score values ranged from -2 to 6 . The area under the curve for the ROC curve was $0.83(95 \%$ CI $0.74-0.90)$. A score of value 0 or less was associated with a sensitivity of $93 \%$ and a score of more than 4 points was associated with a specificity of $92 \%$ for SNPT. Fifty-two point twenty-nine percent of patients had scores of less than one or more than four, what provided strong evidence against and in favor, respectively, for the diagnosis of SNPT. The score developed is a cheap and useful clinical tool for the diagnosis of SNPT and can be used to help therapeutic decisions in patients with suspicion of having SNPT.
\end{abstract}

Key-Words: Tuberculosis, pulmonary, regression analysis, scoring methods.

Tuberculosis is an important public health problem worldwide, which has gained even more importance due to the human immunodeficiency virus (HIV) epidemic and the parallel increase in the multidrug-resistant (MDR) cases. Peru has a high incidence of tuberculosis, which was estimated on 141.4 per 100,000 inhabitants in 2001 [1]. Diagnosis of tuberculosis relies basically on acid-fast bacilli (AFB) smears, which have a sensitivity of around $70 \%$ for pulmonary tuberculosis (PT). This means that there are many cases of PT that are not going to be diagnosed by this test, and they are denominated smear-negative pulmonary tuberculosis (SNPT). The National Control Program (NCP) does not support diagnostic tools other than AFB and, for selected cases, culture (whose results take too long to be of any clinical use). This implies an urgent need for the development of useful and cost-effective clinical tools for the patients with negative smears and strong clinical suspicion of PT.

This need is best evidenced by the high rate of SNPT cases. In Peru, its incidence is calculated in $31 \%$ of all cases [1]. SNPT is usually diagnosed based on the clinical picture, complemented with some radiographic and/or laboratory tests, depending on the availability of resources [2].

According to the World Health Organization guidelines, smear-negative pulmonary tuberculosis is currently defined based on at least three negative sputum smears for AFB, radiographic abnormalities consistent with active $\mathrm{PT}$ and no Received on 21 December 2007; revised 22 April 2008.

Address for correspondence: Dr. Alonso Soto. Hospital Nacional Hipolito Unanue. Av. César Vallejo 1390. El Agustino.Lima 10. Lima. Peru. Email: sotosolari@yahoo.com. Funding: Universidad Peruana Cayetano Heredia through the "Fondo Concursable a la Investigación 2001" and Belgian Directorate-General for Development Cooperation (DGDC).

The Brazilian Journal of Infectious Diseases 2008;12(2):128-132. (C) 2008 by The Brazilian Journal of Infectious Diseases and Contexto Publishing. All rights reserved. response to a course of broad spectrum antibiotics [3]. However, this operational definition does not define the symptoms and radiographic abnormalities are also not clearly specified. The International Society against tuberculosis and lung disease has published guidelines for treatment of tuberculosis in limited resources settings [4], but does not provide a framework for the diagnostic work-up. Also, the guidelines published by the American Thoracic society have very vague suggestions for these cases [5]. In a recent review of SNPT [2], no well validated studies were found in the literature reviewed. So it could be said that currently there are no consensus on the definition for the diagnosis of SNPT, it depends on each clinician's criterion.

A clinical prediction rule (CPR) is defined as a "decisionmaking tool for clinicians that includes three or more variables obtained from the history, or physical examination of the patient, or from simple diagnostic tests and that either provides the probability of an outcome or suggest a diagnostic or therapeutic course of action" [6]. Given the lack of resources to use sophisticated laboratory tests for this problem in our country, we tried to develop a CPR to diagnose SNPT. The objective of the study was therefore to develop it in the format of a score based on simple clinical variables for the diagnosis of SNPT in an area of high endemicity.

\section{Material and Methods}

Setting

The study was done in the emergency department of Cayetano Heredia Hospital, a tertiary level hospital with a catchment population of around 2 million of inhabitants in Lima, Peru, a city with a high incidence of tuberculosis.

Patients

We prospectively included all patients admitted to the Emergency department with clinical suspicion of pulmonary 
tuberculosis, defined as the presence of cough for at least 1 week plus one or more of the following: fever $\left(38^{\circ} \mathrm{C}\right)$, weight loss (4 kg or more in a month), constitutional symptoms (malaise or hyporexia for a minimum of 2 weeks) or dyspnea of any duration. Patients with positive AFB smears were excluded.

Demographic, clinical and radiological data were obtained for all participants within 48 hours of admission. The clinical variables included were age, gender, presence of cough, fever, night sweating, hemoptysis, sputum production, hyporexia, weight loss, dyspnea, chest pain, past history of tuberculosis, a close contact under TB treatment, corticotherapy, alcohol consumption, drug consumption and the presence of comorbidities (such as diabetes mellitus, gastrectomy, chronic renal failure or cancer). The radiological variables included were the presence of any of the following radiological patterns: diffuse reticular, basal infiltrate, upper/apical infiltrate, miliary, cavitary, atelectasis and pleural effusion. For every patient, 2 sputum smears for Ziehl-Neelsen staining and a culture for Mycobacterium tuberculosis in Ogawa media were done. HIV ELISA test was performed after voluntary counseling and testing. For the purposes of the study, PT was defined as a positive culture in Ogawa media. As we excluded the patients with smear-positive tuberculosis, finally we had 2 groups: culture positive SNPT and culture-negative patients. The study was approved by the hospital ethical committee. An informed consent was obtained for all patients included.

\section{Statistical Analysis}

We developed a score based on a multivariate logistic regression model. As a first step, a univariate logistic regression analysis was done. All the variables with a significance level of less than 0.25 were included in the multivariate model. Correlation between variables was studied, and if 2 variables had strong correlation (more than 0.5 ) then the most relevant variable (in terms of Statistical and/or clinical significance) was kept in order to avoid multicollinearity. The model was reduced using backward elimination of nonsignificant variables. The final model included those variables with $\mathrm{p}$ values of less than 0.05 . The numerical values of the score developed were based on the $\beta$-coefficients obtained from the regression model. Goodness of fit was evaluated using the Hosmer and Lemeshow test. The values obtained from the score developed were regressed using bootstrapping against the variables employed, in order to evaluate the internal validity of the results obtained. All analysis were done using SAS v8, SPSS v11 and S-plus v6.

\section{Results}

345 patients were admitted to the emergency room from August 2002 to August 2003 with respiratory symptoms, out of them 83 (24.0\%) were smear-positive TB. 262 subjects met the inclusion criteria for our study and were included in the final analysis. Out if these, 27 (10.31\%) were diagnosed with SNPT. Baseline characteristics of the population as well as radiological findings are shown in Tables 1 and 2. 6 subjects refused HIV testing. In general, the most common causes for going to the hospital were fever and hemoptysis. It is also noteworthy that a third of the patients had previous history of PT.

The results of the score based on the multivariate logistic regression model are shown in Table 3. These values are based on the final multivariable regression model. The goodness of fit of the model was "good" as judged by the Hosmer and Lemeshow statistic (9.23 based on 8 degrees of freedom, p value $=0.32$ ).

The score ranged between -2 and 10 points, nevertheless, in our population the maximum score obtained was 7 . The Area under the curve in the ROC curve was 0.83 (95\% CI 0.740.90 ) indicating a good performance of the score (Figure 1). A cut-off point of 2 gives the best result in terms of combined sensitivity (70\%) and specificity (82\%). A cut-off point of 0 is associated with a sensitivity of $93 \%$. On the other hand, the presence of a score of more than 4 points is associated with a specificity of $92 \%$. Table 4 shows the different values of sensitivities and specificities associated which each possible cut-off point. The bootstrapping regression of the score on the variables included showed highly significant $\mathrm{p}$ values $(p<0.01)$ for all the variables present in the score, with the same coefficients as the previously obtained, indicating that the internal validity of the score was appropriate.

\section{Discussion}

The development of improved and affordable diagnostic tools and efficient strategies for the diagnosis of pulmonary smear negative and extrapulmonary tuberculosis represent areas of priority in public health research, especially for limitedresources settings. The main aim of our study was to prospectively develop a clinical prediction rule that could be useful for improving the diagnostic approach to the patient with clinical suspicion of SNPT.

We used logistic regression to develop our score. Other alternatives would have been the use of other regression models for binary variables or to perform an algorithmic approach using, for example, classification and regression trees (CART). However, the widespread availability and comprehensiveness of logistic regression techniques and the simplicity of presenting the results as simple scores have conducted us to this election.

Two aspects we would like to comment are the presence of an age of more than 45 years and the presence of productive cough as factors associated with a lower probability of having SNPT. Advanced age is normally associated with a higher susceptibility to tuberculous disease. However, in developing countries, tuberculosis is predominantly a disease of young people. Old patients with pulmonary complaints have usually a broader spectrum of diseases, which can diminish the performance of the score. The presence of productive cough can be associated with a better yield of AFB smear, so it is not surprising that it is negatively associated with smear-negative

www.bjid.com.br 
Table 1. Sociodemographic and clinical characteristics of the study population

\begin{tabular}{lccr}
\hline & $\begin{array}{c}\text { Culture positive } \\
(\mathbf{N}=\mathbf{2 7})^{*}\end{array}$ & $\begin{array}{c}\text { Culture negative } \\
\text { (N=235)* }\end{array}$ & pvalue† \\
\hline Male Sex & $19(70.37)$ & $147(62.55)$ & 0.28 \\
Age more than 45 years & $21(77.77)$ & $111(47.23)$ & 0.01 \\
Cough & $25(92.59)$ & $220(87.64)$ & 0.54 \\
Fever & $14(51.85)$ & $113(48.09)$ & 0.43 \\
Hemoptysis & $20(74.07)$ & $102(43.40)$ & $<0.01$ \\
Productive cough & $16(59.26)$ & $194(82.55)$ & $<0.01$ \\
Weight loss & $19(70.37)$ & $140(59.57)$ & 0.19 \\
Dyspnea & $13(48.15)$ & $135(57.45)$ & 0.24 \\
Thoracic pain & $11(40.74)$ & $98(41.70)$ & 0.55 \\
History of Tuberculosis & $8(29.63)$ & $86(36.60)$ & 0.31 \\
Tuberculous contact & $10(37.04)$ & $85(37.45)$ & 0.88 \\
HIV infection $\ddagger$ & $3(11.11)$ & $25(10.92)$ & 0.70 \\
Alcoholism & $4(14.81)$ & $47(20.09)$ & 0.76 \\
\hline
\end{tabular}

*Percentages are shown between parentheses. $\dagger \mathrm{p}$ values in the initial univariate regression model. $\ddagger 6$ patients refused testing (all of them were culture negative).

Table 2. Radiological characteristics of the study population

\begin{tabular}{lccc}
\hline & $\begin{array}{c}\text { Culture positive } \\
(\mathbf{N = 2 7 ) *}\end{array}$ & $\begin{array}{c}\text { Culture negative } \\
(\mathbf{N}=\mathbf{2 3 5})^{*}\end{array}$ & pvalue† \\
\hline Interstitial infiltrate & $8(29.63)$ & $98(41.70)$ & 0.16 \\
Cavitation & $10(37.04)$ & $54(22.98)$ & 0.09 \\
Miliary infiltrate & $3(11.11)$ & $6(2.55)$ & 0.05 \\
Alveolar infiltrate & $14(51.85)$ & $100(42.55)$ & 0.24 \\
Pneumothorax & $1(3.7)$ & $7(3.70)$ & 0.59 \\
Pleural effusion & $0(0)$ & $15(6.38)$ & 0.19 \\
Atelectasis & $0(0)$ & $10(4.26)$ & 0.33 \\
Apical infiltrate & $14(51.85)$ & $43(18.30)$ & $<0.01$ \\
\hline
\end{tabular}

*Percentages are shown between parentheses. $\dagger \mathrm{p}$ values based on the initial univariate regression model.

Table 3. Predictors of TB in smear negative patients based on the results of multiple logistic regression analysis

\begin{tabular}{lccc}
\hline Predictors & $\begin{array}{c}\text { Multivariate OR } \\
\text { (95\% CI) }\end{array}$ & p value & Score weight \\
\hline Clinical & $3.24(1.11-9.22)$ & 0.03 & 2 \\
$\quad$ Hemoptysis & $2.35(0.86-6.43)$ & 0.09 & 1 \\
$\quad$ Weight loss & $2.01(1.01-3.01)$ & $<0.01$ & -1 \\
$\quad$ Age more than 45 years & $0.35(0.14-0.90)$ & 0.03 & -1 \\
$\quad$ Expectoration & & & \\
Radiographic (chest X-ray) & $4.29(1.70-10.86)$ & $<0.01$ & 3 \\
$\quad$ Apical infiltrate & $9.31(2.21-39.24)$ & $<0.01$ & 4 \\
$\quad$ Miliary Infiltrate & & \\
\hline
\end{tabular}

pulmonary tuberculosis. In other words, people from which a good sputum sample cannot be obtained, will have a lower probability of having a positive AFB smear. This negative association with SNPT was also found in other studies $[7,8]$. A cut-off point of 0 is associated with sensitivity of $93 \%$ and a specificity of $50 \%$. So the presence of any positive score will be a useful argument for a more in-depth analysis. On the other hand, the presence of a score of more than 4 points is associated with a specificity of 92\%, giving a fairly high probability of PT. So instead of choosing a single cutt-off point to be used, which we consider not accurate, for the application of this model we propose the use of three categories based on the numeric value of the score. In this way, we can consider a score of less or equal than 0 as corresponding to a very low probability of SNPT while scores of more than 4 can be considered as representing a high 
Table 4. Sensitivity, specificity and likelihood ratios for different scores among patients with and without tuberculosis

\begin{tabular}{cccccc}
\hline Score & N & Sensitivity & Specificity & Positive LR & Negative LR \\
\hline-2 & 16 & 1.00 & 0.07 & 1.07 & 0.00 \\
-1 & 55 & 0.96 & 0.30 & 1.37 & 0.12 \\
0 & 43 & 0.93 & 0.48 & 1.77 & 0.15 \\
1 & 36 & 0.85 & 0.62 & 2.25 & 0.24 \\
2 & 50 & 0.70 & 0.82 & 3.85 & 0.36 \\
3 & 21 & 0.48 & 0.88 & 4.04 & 0.59 \\
4 & 18 & 0.33 & 0.92 & 4.16 & 0.73 \\
5 & 16 & 0.19 & 0.99 & 20.55 & 0.73 \\
6 & 7 & 0.00 & 1.00 & $\infty$ & 1 \\
\hline
\end{tabular}

The values for sensitivity, specificity and likelihood ratios are based on a score higher than the cut-off point specified. For example a score of more than 0 will have a sensitivity of 0.93 and a specificity of 0.48. LR=Likelihood Ratio.

Figure 1. Receiver Operator Characteristic (ROC) curve for the score developed.

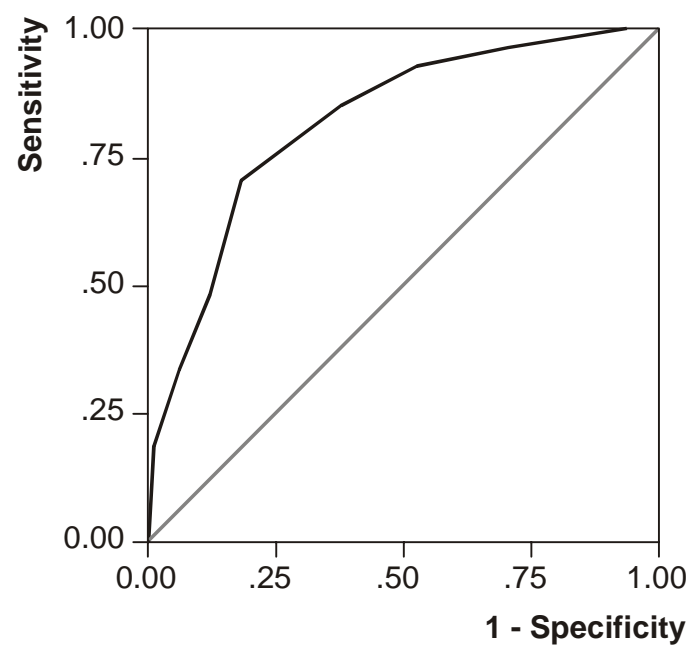

probability of SNPT. Results between 1 and 4 points must be judged as non conclusive and merit further diagnostic evaluation.

One of the most important contributions of our study is the fact that, in more than a half of the patients, we could go for a specific clinical decision based on the results of the score. In other words, in $52.29 \%$ of patients, the score could be considered a strong evidence for ruling out the diagnosis, or initiating anti-TB treatment while pending culture results, especially for severe disease.

Our study has some limitations. First of all, our study population was not the ideal because we only included population in an emergency setting from an area with high prevalence of tuberculosis. The internal validity of the score was judged as appropriate because of the prospective nature of the data collection and the results of the bootstrapping validation. However, the external validity of the score, that is, its performance in other settings such as non-emergency settings or areas with low prevalence of tuberculosis, has to be evaluated in future studies.
This model was developed based on culture as gold standard. We chose it as the gold standard because it is widely accepted and recommended for the diagnosis of smear negative pulmonary tuberculosis. But we must take on account that its sensitivity is relatively low, compared for example with new culture-media or with clinical outcome. In the ideal situation, the criteria for diagnosis of SNPT should include other clinical and laboratory parameters (for example clinical evolution of patients and/or demonstration of AFB or caseating granuloma in histopatological specimens).

The developed score did not include other clinical tools that have been employed for the diagnosis of SNPT such as fibrobroncoscopy [9-13], the use of induced sputum [14-20], concentration techniques [21-24], auramine-rhodamine enhanced AFB detection [25,26], or Adenosine deaminase activity in sputum or bronchoalveolar lavage [27-30] or fine needle aspiration $[31,32]$. Also, the use of liquid media culture was not evaluated. All of these additional diagnostic tests merit further evaluation and the added value of these tests with respect to the developed score should also be a field for future research. However, we intended to develop a clinical tool for resource-limited settings, where these expensive tools are not available.

Our results suggest that the score developed can be a useful tool, not a rule, for the diagnosis of smear negative tuberculosis and can give information that can lead to a clinical decision in around half of the patients evaluated. This can reduce the number of unnecessary procedures done (for example, in patients with a negative score and therefore a very low probability of SNPT) and can be used to avoid the delay in treatment for those patients with high scores while pending other tests. It is not the intention to replace the clinical judgment by this score, its best use, as well as the one of other clinical predictions rules, is as supplementary information that can help to take a decision during the evaluation of a patient while facing a patient with clinical suspicion of smear negative pulmonary tuberculosis.

In conclusion, the score developed represents an inexpensive and useful tool for the diagnosis of SNPT in the 
population studied. Future lines of research will include the score validation in another settings and/or including the score in clinical diagnostic algorithms.

\section{Acknowledgements}

Our acknowledgements to Universidad Peruana Cayetano Heredia for financing the recruitment of patients and laboratory tests; to Freddy Perez for recruiting the patients in the Emergency Room; to the Institute of Tropical Medicine Alexander von Humboldt (UPCH) and Belgian cooperation (DGDC) for their colaboration.

\section{References}

1. Programa Nacional de Control de la Tuberculosis. Actualización De la Doctrina, normas y procedimientos para el control de la tuberculosis en el Perú. Lima: Ministerio de Salud; 2001.

2. Siddiqi K., Lambert M.L., Walley J. Clinical diagnosis of smearnegative pulmonary tuberculosis in low-income countries: the current evidence. Lancet Infect Dis 2003;3(5):288-96.

3. WHO. Treatment of tuberculosis. Guidelines for National Programmes. Geneva; WHO; 2003 (WHO/CDS/TB 2003.313).

4. Enarson D.A., Rieder H.L., Arnadottir T., Trébucq A. Management of tuberculosis. A guidefor low income countries. 2000. Fifth Edition. 5 ed. Compogravure Impression, Broachage Imprimerie, St-Just-La-Pendue.

5. Diagnostic Standards and Classification of Tuberculosis in Adults and Children. Am J Respir Crit Care Med 2000;161:1376-95.

6. Laupacis A., Sekar N., Stiell I.G.. Clinical prediction rules. A review and suggested modifications of methodological standards. JAMA 1997;277(6):488-94.

7. Kanaya A.M., Glidden D.V., Chambers H.F. Identifying pulmonary tuberculosis in patients with negative sputum smear results. Chest 2001;120(2):349-55.

8. Samb B., Sow P.S., Kony S., et al. Risk factors for negative sputum acid-fast bacilli smears in pulmonary tuberculosis: results from Dakar, Senegal, a city with low HIV seroprevalence. Int J Tuberc Lung Dis 1999;3(4):330-6.

9. Charoenratanakul S., Dejsomritrutai W., Chaiprasert A. Diagnostic role of fiberoptic bronchoscopy in suspected smear negative pulmonary tuberculosis. Respir Med 1995;89(9):621-3.

10. Chawla R., Pant K., Jaggi O.P., et al. Fibreoptic bronchoscopy in smear-negative pulmonary tuberculosis. Eur Respir J 1988;1(9):804-6.

11. McWilliams T., Wells A.U., Harrison A.C., et al. Induced sputum and bronchoscopy in the diagnosis of pulmonary tuberculosis. Thorax 2002;57(12):1010-4.

12. Watanabe K., Inoue Y., Shimoda T., et al. [Diagnostic usefulness of transbronchial aspiration and bronchial lavage for pulmonary tuberculosis]. Kekkaku 1990;65(3):227-30.(abstract)

13. Yuksekol I., Bal S., Ozkan M., et al. [The value of fiberoptic bronchoscopy in diagnosis of smear negative pulmonary tuberculosis]. Tuberk Toraks 2003;51(4):405-9.

14. Al Zahrani K., Al Jahdali H., Poirier L., et al. Yield of smear, culture and amplification tests from repeated sputum induction for the diagnosis of pulmonary tuberculosis. Int $\mathrm{J}$ Tuberc Lung Dis 2001;5(9):855-60.
15. Anderson C., Inhaber N., Menzies D. Comparison of sputum induction with fiber-optic bronchoscopy in the diagnosis of tuberculosis. Am J Respir Crit Care Med 1995;152(5 Pt 1):1570-4.

16. Conde M.B., Soares S.L., Mello F.C., et al. Comparison of sputum induction with fiberoptic bronchoscopy in the diagnosis of tuberculosis: experience at an acquired immune deficiency syndrome reference center in Rio de Janeiro, Brazil. Am J Respir Crit Care Med 2000;162(6):2238-40.

17. Hartung T.K., Maulu A., Nash J., Fredlund V.G. Suspected pulmonary tuberculosis in rural South Africa--sputum induction as a simple diagnostic tool? S Afr Med J 2002;92(6):455-8.

18. Li L.M., Bai L.Q., Yang H.L., et al. Sputum induction to improve the diagnostic yield in patients with suspected pulmonary tuberculosis. Int J Tuberc Lung Dis 1999;3(12):1137-9.

19. Parry C.M., Kamoto O., Harries A.D., et al. The use of sputum induction for establishing a diagnosis in patients with suspected pulmonary tuberculosis in Malawi. Tuber Lung Dis 1995;76(1):72-6.

20. Shata A.M., Coulter J.B., Parry C.M., et al. Sputum induction for the diagnosis of tuberculosis. Arch Dis Child 1996;74(6):535-7.

21. Apers L., Mutsvangwa J., Magwenzi J., et al. A comparison of direct microscopy, the concentration method and the Mycobacteria Growth Indicator Tube for the examination of sputum for acidfast bacilli. Int J Tuberc Lung Dis 2003;7(4):376-81.

22. Bruchfeld J., Aderaye G., Palme I.B., et al. Sputum concentration improves diagnosis of tuberculosis in a setting with a high prevalence of HIV. Trans R Soc Trop Med Hyg 2000;94(6):677-80.

23. Wilkinson D., Sturm A.W. Diagnosing tuberculosis in a resourcepoor setting: the value of sputum concentration. Trans $\mathrm{R}$ Soc Trop Med Hyg 1997;91(4):420-1.

24. Woods G.L., Pentony E., Boxley M.J., Gatson A.M. Concentration of sputum by cytocentrifugation for preparation of smears for detection of acid-fast bacilli does not increase sensitivity of the fluorochrome stain. J Clin Microbiol 1995;33(7):1915-6.

25. Strumpf I.J., Tsang A.Y., Sayre J.W. Re-evaluation of sputum staining for the diagnosis of pulmonary tuberculosis. Am Rev Respir Dis 1979;119(4):599-602.

26. Selvakumar N., Sudhamathi S., Duraipandian M., et al. Reduced detection by Ziehl-Neelsen method of acid-fast bacilli in sputum samples preserved in cetylpyridinium chloride solution. Int $\mathrm{J}$ Tuberc Lung Dis 2004;8(2):248-52.

27. Lakshmi V., Rao R.R., Joshi N., Rao P.N. Serum adenosine deaminase activity in bacillary or paucibacillary pulmonary tuberculosis. Indian J Pathol Microbiol 1992;35(1):48-52.

28. Orphanidou D., Stratakos G., Rasidakis A., et al. Adenosine deaminase activity and lysozyme levels in bronchoalveolar lavage fluid in patients with pulmonary tuberculosis. Int J Tuberc Lung Dis 1998;2(2):147-52.

29. Dilmac A., Ucoluk G.O., Ugurman F., et al. The diagnostic value of adenosine deaminase activity in sputum in pulmonary tuberculosis. Respir Med 2002;96(8):632-4.

30. Kayacan O., Karnak D., Delibalta M., et al. Adenosine deaminase activity in bronchoalveolar lavage in Turkish patients with smear negative pulmonary tuberculosis. Respir Med 2002;96(7):536-41.

31. Gomes I., Trindade E., Vidal O., Yet al. Diagnosis of sputum smearnegative forms of pulmonary tuberculosis by transthoracic fineneedle aspiration. Tubercle 1991;72(3):210-3.

32. Yuan A., Yang P.C., Chang D.B., et al. Ultrasound guided aspiration biopsy for pulmonary tuberculosis with unusual radiographic appearances. Thorax 1993;48(2):167-70. 